\title{
L'information dans les bibliothèques publiques comme nouvel instrument de marketing
}

Information in public libraries viewed as a new marketing instrument

\section{La información en las bibliotecas públicas un nuevo instrumento del marketing}

\section{Réjean Savard}

Volume 29, numéro 4, octobre-décembre 1983

URI : https://id.erudit.org/iderudit/1053607ar

DOI : https://doi.org/10.7202/1053607ar

Aller au sommaire du numéro

Éditeur(s)

Association pour l'avancement des sciences et des techniques de la documentation (ASTED)

ISSN

0315-2340 (imprimé)

2291-8949 (numérique)

Découvrir la revue

Citer cet article

Savard, R. (1983). L'information dans les bibliothèques publiques comme nouvel instrument de marketing. Documentation et bibliothèques, 29(4),

135-141. https://doi.org/10.7202/1053607ar
Résumé de l'article

Les différents éléments du problème sont présentés et définis : information, besoins d'information, types de besoin. Il est démontré ensuite comment la bibliothèque publique à l'extérieur du Québec s'est développée comme centre d'information. Les possibilités d'implanter ce concept au Québec sont envisagées.
Tous droits réservés (c) Association pour l'avancement des sciences et des techniques de la documentation (ASTED), 1983
Ce document est protégé par la loi sur le droit d'auteur. L'utilisation des services d'Érudit (y compris la reproduction) est assujettie à sa politique d'utilisation que vous pouvez consulter en ligne. 


\title{
L'information dans les bibliothèques publiques comme nouvel instrument de marketing ${ }^{1}$
}

\author{
Réjean Savard* \\ École de bibliothéconomie \\ Université de Montréal
}

Les différents éléments du problème sont présentés et définis: information, besoins d'information. types de besoin. /l est démontré ensuite comment la bibliothèque publique à l'extérieur du Québec s'est développée comme centre d'information. Les possibilités d'implanter ce concept au Québec sont envisagées.

\section{Information in public libraries viewed as a new marketing instrument}

The various elements of the problems are submitted and defined: information, need for information, types of needs. It is then showed how the public library outside Quebec has grown as an information center. The possibilities of implementing this concept in Quebec are then examined.
La información en las bibliotecas públicas un nuevo instrumento del marketing

Los distintos elementos del problema se presentan y se definen: información, necesidades de información, tipos de necesidades. Se muestra a continuación de qué forma la biblioteca pública a las afueras de Quebec se ha desarrollado como centro de información. Se contempla la posibilidad de implantar este concepto en Quebec.
Le monde des bibliothèques publiques au Québec a franchi ces derniers temps des étapes importantes. En quelques années seulement, on a effectué des pas de géants en ce qui concerne notamment la construction de nouvelles bibliothèques, grâce à ce qu'il est convenu d'appeler le "plan Vaugeois». Plusieurs municipalités ont en effet profité des subventions considérables mises à leur disposition par le gouvernement du Québec pour doter leurs citoyens d'édifices convenables afin d'abriter leur bibliothèque. II faut certes louanger le ministère des Affaires culturelles et ses fonctionnaires pour avoir réalisé un programme de si grande envergure. Grâce à eux. les bibliothèques publiques du Québec sont maintenant en meilleure posture qu'ily a cinq ans alors que le ministre Vaugeois lui-même constatait, lors d'un discours au Congrès de I'ASTED. leur piètre situation en comparaison avec les bibliothèques publiques de l'Ontario².
Cependant, il reste beaucoup à faire sur d'autres plans et il ne faudrait pas que les artisans de ces victoires des dernières années s'assoient sur leurs lauriers. On a en effet démontré qu'en moyenne, seulement $26.65 \%$ des gens au Québec avaient déjà utilisé au moins une fois leur bibliothèque publique par le passé et que ce pourcentage était infiniment plus réduit lorsune orientation définitivement axée sur la satisqu'on parlait d'utilisation "relativement» fréquente (une fois par mois) ${ }^{3}$. La « rentabilité« des bibliothèques publiques est donc encore loin d'être atteinte et il faudra accroître encore plus leur visibilité si celles-ci veulent continuer de recevoir l'appui financier des contribuables.

C'est dans cet esprit, en partie du moins, que s'est développé dans d'autres états le concept des bibliothèques publiques comme centre d'information ("community information services»).
- L'auteur est professeur à l'École de bibliothéconomie.

1 Version revisée d'une communication présentée en octobre 1980 dans le cadre des conférences Aegidius-Fauteux.
2. "Discours du ministre des affaires culturelles. M. Denis Vaugeois, au 5 e congrès de l'ASTED», Bıblio-contact, vol. 4. no 3 (1978), 2-5.

3. Réjean Savard et Jean Cobein, «L'utilisation des bibliothèques publiques au Québec en regard de certaines variables endogènes». Argus, vol. 11. 1 (1982), 3-8. 
Plusieurs auteurs ont déjà expliqué en détails ce concept, notamment auxÉtats-Unis ${ }^{4}$ et en GrandeBretagne $^{5}$. II s'agit en fait d'un concept relativement nouveau qui. lorsque mis en valeur par la bibliothèque publique, élargit sensiblement le cadre de sa mission. Ainsi, d'un concept statique qui s'apparente davantage à un «self-service de la lecture-loisirs», la bibliothèque passe à un concept dynamique de véritable "service d'information documentaire». II en résulte une responsabilité sociale accrue, plus d'usagers et par conséquent, plus de possibilités de subventions.

Le présent article vise à présenter les principaux éléments de ce nouveau concept et à en discuter les possibilités d'implantation, au Québec, dans une optique "marketing», c'est-àdire une orientation définitivement axée sur la satisfaction des besoins de la clientèle actuelle et potentielle. ${ }^{6}$

\section{L'information, c'est quoi?}

Comme le souligne Wilcox, l'impact de l'information dans la société moderne est certainement des plus importants: elle joue un rôle essentiel en éducation, supporte toute la recherche nécessaire à une vie saine et prospère, améliore la qualité de la vie et son apport est déterminant dans le fonctionnement politique des sociétés ${ }^{7}$.

Robin Crickman ${ }^{8}$ explique comment se vérifie la valeur de l'information en se servant d'un exemple tout à fait concret: le chemin de fer. On ne peut pas, dit-il, laisser circuler anarchiquement les trains sur la voie ferrée: il en résulterait des télescopages et des carambolages tragiques. Pour éviter ces incidents, il est essentiel d'obtenir le plus d'informations possibles concernant la localisation des trains sur la voie. Plus on aura d'informations, plus on pourra laisser les trains rouler plus proches les uns des autres. Il en résultera une meilleure utilisation des équipements et une rationalisation des services qui profiteront à la compagnie de chemin de fer bien sûr. mais aussi à toute la société. Car le fait de produire le double de ce qui était auparavant possible de produire avec les mêmes ressources, et ce grâce à l'information, profite globalement à toute la société.

En fait, nous vivons dans une société d'information. Qui parmi nous n'a pas constaté l'éclatement qui a caractérisé les dernières décen- nies? Nous sommes débordés, ensevelis sous des montagnes d'informations. Les «services» d'information se multiplient. Comment alors l'information peut-elle devenir un problème? Comment peut-on manquer d'information? La réponse est simple: la société a plus ou moins perdu le contrôle de cette information. L'information n'arrive pas au moment où elle devrait arriver. L'information est introuvable. L'information n'est pas fournie à la bonne personne ou elle est inégalement distribuée. Obtenir la bonne information au bon moment peut devenir un défi difficile à relever. Le fossé qui existe entre l'information et ceux qui la cherchent, s'élargit de plus en plus en même temps qu'il devient plus évident.

Même si on parle souvent d'information aujourd'hui et qu'on semble bien en connaitre les effets et les mérites, peu de gens sont capables de définir clairement ce concept. Les «scientistes de l'information» eux-mêmes en sont rarement venusà s'entendre sur la signification de ce $\operatorname{mot}^{9}$.

D'après l'étude dirigée par Kahn sur les centres d'information communautaires, la fonction d'information peut être prise dans un sens très large. c'est-à-dire qu'elle comprend même, d'après lui, le fait de conseiller une personne socialement dépourvue ${ }^{10}$. Warner est également d'avis qu'informer et conseiller sont parfois très près l'un de l'autre ${ }^{11}$. Childers, le bibliothécaire qui s'est peutêtre le plus intéressé aux besoins d'information. distingue entre apprendre et s'informer. Ce sont là deux choses différentes, écrit-il, puisque l'information est une donnée de base décrite comme le matériel utilisé pour connaître, prendre des décisions, agir, penser et finalement apprendre ${ }^{12}$. Il distingue également entre information et connaissance, cette dernière se rattachant à un individu, un "connaissant », ce qui n'est pas le cas pour l'information qui est tout à fait «impersonnelle».

Sylvia Faibisoff et Donald Ely, dans un état de la question sur l'information et les besoins en information s'attardent davantage à distinguer données (data) et information. Les données (data) sont du matériel brut qui servent à former ou à fournir l'information. Ce que nous appelons souvent information, écrivent-ils, c'est une collection de données prises au hasard qui ne deviennent information que lorsque utilisées dans un
4. Clara S. Jones, Public library information and referral service. Syracuse, N.Y., Gaybord Professionnal Publications. 1978. $265 \mathrm{p}$

5. Allan Bunch. Community information services: their orıgin. scope and development. London. Clive Bingley. 1982. $168 \mathrm{p}$

6. Benedict Leeburger. Marketıng the /ibrary, White Plains, N.Y., Knowledge Industry Publications, 1982, p. 5-6. (Professional Librarian Series).

7. A.E. Wilcox, «Library cooperative relationships in connection with emerging service patterns", Library Trends, vol. 28. no. 2 (1979). 329-330
8. Robın D. Crickman. "The emerging information professıonal". Library Trends, vol. 28, no. 2 (1979). 311-328.

9. Hans Wellisch, «From information science to informatıcs: a termınological investigation". Journal of Librarıanship. vol. 4. no. 3 (1972), 157-187

10. A.J. Kahn et al.. Neıghbourhood informatıon centers. New York. Columbia University School of Social Work. 1966. p. 33-34

11 Edgar S. Warner. Information needs of urban residents. Washıngton, U.S., Department of Health. Educatıon and Welfare. 1973. p. 24-25

12. Thomas Childers. The information-poor in America. Metu chen, N.J., Scarecrow Press, 1975. p. 13 
but bien spécifique. L'information est une représentation symbolique de la réalité et sa caractéristique principale est sa "capacité de réduire l'incertitude". Ce discours les amène à une définition que nous adopterons pour notre exposé: "information is a symbol or a set of symbols which has the potential for meaning ${ }^{13}$.

\section{Tableau 1}

\section{Continuum des terminologies de l'information}
1. Les données
2. L'information
3. La connaissance
4. L'appris

En utilisant cette définition et les autres éléments mentionnés par les différents auteurs, on peut imaginer un continuum allant des données brutes à l'« appris»: le dernier niveau est celui où les données [1] ont été d'abord organisées pour former une information [2], puis où cette même information a été rattachée à un individu, un "connaissant» [3] et enfin, où cette connaissance a été assimilée par un individu, mûrie et où elle est prête à être réexprimée à nouveau. c'està-dire apprise [4].

\section{Besoin d'information}

Maslow s'est rendu célèbre par ses études sur les besoins. II démontre que ceux-ci sont généralement «hiérarchisés» ${ }^{14}$. Son approche n'est pas étrangère à celle utilisée par ceux qui ont étudié les besoins d'information. En effet, les recherches effectuées dernièrement ont démontré qu'un besoin en information était fort complexe.

Stevenson (1978) explique que les besoins d'information s'apparentent davantage aux beso!ns qu'il appelle de haut niveau (high-level needs) comme les besoins de réussite, de succès et d'auto-réalisation. De plus, ajoute-t-il, les gens ont tendance à percevoir leurs besoins en termes de moyens pour atteindre ceux-ci, ce qui ne facilite en rien leur évaluation ${ }^{15}$.

Les chercheurs se sont d'abord penchés sur la terminologie des besoins. La langue anglaise fournit d'ailleurs plusieurs termes apparentés à ce mot (need). En 1974. Maurice Line qui s'était particulièrement attardé aux besoins d'information scientifique et technique, proposait une série de définitions visant à clarifier la situation ${ }^{16}$. Il définissait successivement "requirement». "use», «demand», «want» et «need». Ce dernier terme représente ce que les gens devraient avoir alors que «want» réfère plutôt à ce que les gens aimeraient avoir. Les deux peuvent, mais pas nécessairement, devenir une "demande» (demand) qui, elle, réfère à ce que les gens demandent effectivement. Quant au terme « requirement», il s'agit d'un terme générique qui couvre les trois définis précédemment. La langue française permet difficilement ces distinctions.

Childers, de son côté, affirme que le mot besoin est un concept tout à fait abstrait développé à partir d'observations du langage ou des agissements des personnes mais qu'il est excessivement difficile de le définir ${ }^{17}$. II faut donc, pour le mesurer, se tourner vers les traces qu'il laisse. c'est-à-dire vers l'expression des besoins de ces personnes. Pour ce faire, on observe ce qu'ils disent, ce qu'ils font et ce qu'ils sont.

Faibisoff et Ely, dans leur état de la question mentionné plus tôt, constatent également cette complexité des besoins en information qu'ils n'arrivent pas à définir. Cependant, une chose est certaine, affirment-ils, les gens ont besoin d'information; tout le monde en a besoin.

Un peu comme Maslow, plusieurs auteurs ont tenté une hiérarchisation des besoins d'information. Le premier à le faire fut probablement Taylor en 1968. Il décrivait un continuum suivant lequel s'expriment les besoins:

1) un besoin réel, mais non exprimé:

2) une description consciente du besoin:

3) une définition formelle du besoin:

4) l'expression du besoin ${ }^{18}$

Il devenait dès lors évident qu'un besoin d'information pouvait être présent chez une personne, tout en n'étant pas conscient.

En 1975. Childers ajoute une nouvelle dimension. II parle de besoins kinétiques et de besoins potentiels. Les besoins kinétiques répondent à une situation présente, s'associent à un problème spécifique ou à une réalité bien particulière. Les besoins potentiels sont plus ou moins bien définis et répondent à des réalités plutôt lointaines. Cependant, les deux types de besoins peuvent être conscients ou inconscients, et à des degrés divers ${ }^{19}$.
13. Sylvia Faibısoff and Donald Ely, «Information and Informatıon needs". Information Reports and Bıbliographies, vol. 5. no. 5 (1976). 3

14. Abraham Maslow. Motivation and personnalıty. New York. Harper and Row, 1975, p. 90-106.

15. G. Stevenson, "The publıc library in a communicatıon settıng». Library Quarterly, vol. 48, no. 4 (1978). 393-415.
16. Maurice B. Line, "Draft definitions", Aslib Proceedings, vol. 26 . no. 2 (1974), 87

17. Thomas Childers, The information-poor.

18. R. Taylor, "Questions-negotiation and Information seeking is 'ibraries", College and Research libraries, vol. 29. no. 3 (May 1968) 178-194.

19. Thomas Childers, The information-poor.. 
L'approche la plus intéressante semble être celle de Barry Totterdell et Jean Bird dans leur étude sur l'efficacité de la bibliothèque publi$q^{20}$. D'après leur recherche, il y aurait trois niveaux d'expression des besoins d'information: le besoin exprimé. le besoin non exprimé. et le besoin non activé. Le besoin exprimé, c'est le besoin conscient d'information, celui qui peut être verbalisé à des degrés divers. II se manifeste généralement par une demande d'information. soit à la bibliothèque ou ailleurs. Le besoin non exprimé est pressenti ou conscient chez la personne mais celle-ci n'arrive pas à l'exprimer convenablement. L'absence d'une institution ou d'un service ou parfois l'ignorance de l'existence de ce service peut effectivement nuire à l'expression de ce type de besoin. Enfin, le besoin non activé d'information est particulièrement difficile à cerner car il "sommeille» chez la personne. II ne s'agit que d'un besoin inconscient d'information: un individu fait face à un problème quelconque et pour différentes raisons, il ne réalise pas qu'obtenir telle information résoudrait ce problème. II peut aussi s'agir d'un barrage psychologique, car avouer ses besoins d'informations, c'est aussi avouer ses faiblesses ou ses carences.

\section{Types de besoins d'information non spécialisés}

On peut se demander maintenant de quelles sortes d'information ont besoin les individus moyens. Plusieurs chercheurs ont abordé la question et ont fourni diverses catégorisations des besoins des usagers à partir desquelles il est possible de généraliser quelque peu21

Le tableau 2 fournit un échantillon de ces catégorisations de besoins d'information non spécialisés. On voit que les catégories les plus souvent mentionnées sont l'éducation et la santé, puis viennent celles de l'emploi, de la consommation. du logement et des problèmes d'ordre légal. Viennent ensuite les problèmes de transports et de loisirs.

Pour obtenir ces informations, les personnes font appel à différentes sources. Beal parle de sources informelles et de sources formelles 22. D'après Warner, les sources informelles sont les

Tableau 2

Catégories de besoins

\begin{tabular}{ll} 
KAHN (1966) & WARNER (1973) \\
1. communication & 1. communauté \\
et voyages & 2. consommation \\
2. éducation & 3. logement et entretien \\
et formation & \multicolumn{1}{c}{ ménager } \\
3. emploi & 4. criminalité et sécurité \\
4. informations unique. & 5. éducation \\
locale et nationale & 6. emploi \\
5. informations person- & 7. transports \\
nelle et familiale & 8. santé \\
6. assurance & 9. divers \\
7. santé et médecine & 10. loisirs \\
8. propriétés et terrains & 11. discrimination \\
9. service militaire et & 12. problèmes financiers \\
anciens combattants & 13. problèmes légaux \\
10. commerce et industrie & 14. assistance publique
\end{tabular}

CHILDERS (1975)
1. santé
2. problèmes familiaux
et ménagers
3. consommation
4. logement
5. emploi
6. bien-être social
7. droit
8. information politique
9. transports
10. éducation
11. loisirs

TURICK (1978)
1. carrière
2. enfants
3. organisations
religieuses
4. communauté
5. consommation
6. culture
7. éducation
8. urgence
9. environnement
10. gouvernement
11. santé
12. hôpitaux
13. logement
14. droit
15. santé mentale
16. alimentation
17. loisirs
18. réhabilitation
19. sécurité
20. sécurité sociale
21. services sociaux
22. âge d'or
23. magasins
d'escompte
24. transports
25. carrières
26. bien-être social

20. Barry Totterdell and Jean Bird, The effective library, London, Library Association, 1976. 207 p.

21. A.J. Kahn et al., Neighbourhood information...; Edgar S. Warner, Information needs... ; Thomas Childers. The infor- mation-poor...: Dorothy Turick, Community information services in libraries. New York, Bowker, 1978, 80 p. (Library journal special report no. 5).

22. Christina Beal, «Studying the public's information needs", Journal of Librarianship. vol. 11. no. 2 (1979). 130-151. 
plus utilisées ${ }^{23}$. Un besoin d'information est comblé par une source informelle lorsque la personne explique son problème soit à un parent, à un ami ou à un voisin. Comme le souligne également Beal, ce peut être aussi le médecin de famille, le gérant de banque, un travailleur social. un agent de police ou un ministre du culte. Ces individus deviennent des interlocuteurs privilégiés et les sources formelles, comme les différents services d'information gouvernementaux. les bibliothèques et autres centres d'information. sont utilisés en dernier recours seulement. Warner souligne même que parmi les nombreux répondants à sa recherche sur les besoins d'information, seulement $3 \%$ déclaraient avoir utilisé une bibliothèque pour obtenir une réponse à leurs besoins d'information.

\section{La bibliothèque publique comme centre populaire d'information}

Dans plusieurs pays, que ce soit en Scandinavie, en Angleterre ou aux États-Unis, on accorde de plus en plus d'importance à l'information dans les bibliothèques publiques. Les premières expériences de «community information centers » dans les bibliothèques publiques peuvent être retracées il y a plusieurs années. C'est au début des années 1970 qu'on s'est vraiment intéressé au problème. II semble que ce soit la conférence organisée en 1971 par l'Université d'Illinois et intitulée «Libraries and neighbourhood information centers" qui ait déclenché ce nouvel intérêt chez les bibliothécaires ${ }^{24}$. En effet, plusieurs bibliothèques publiques, grandes et petites, ont par la suite développé un service semblable. Une des premières fut d'ailleurs la petite bibliothèque publique de Dade County en Floride. Le projet le plus ambitieux fut sans doute celui patronné par Thomas Childers dans les bibliothèques publiques de cinq grandes villes américaines: Atlanta. Cleveland. Détroit. Houston et Queens Borough (New York). L'expérience de Détroit est peut-être celle dont on a parlé le plus. Communément appelé "TIP» (The information place), elle fut mise en place par la directrice des bibliothèques publiques. Clara Stanton Jones. Celle-ci, une bibliothécaire de race noire au dynamisme percutant, avait longtemps été en poste dans les petites succursales des quartiers défavorisés de Détroit et connaissait les multiples problèmes auxquels doit faire face la population des ghettos américains. On trouve plusieurs comptes rendus de cette expérience dans la littérature professionnelle 25
Plus près de nous, dans la province ontarienne. on annonçait assez récemment que les services d'information communautaires du ministère de la Culture et de la Récréation allaient être regroupés sous la division des bibliothèques publiques qui s'appellera désormais "Division des bibliothèques et de l'information communautaire" (Libraries and community information branch) ${ }^{26}$. Cette décision fait suite à une recommandation de la fameuse enquête sur les bibliothèques publiques en Ontario qui recommandait au gouvernement ontarien de faciliter la mise sur pied de services d'information communautaires dans les bibliothèques publiques, notamment par l'attribution d'octrois ${ }^{27}$. Le rapport Bowron signalait qu'il y a de nombreux avantages à utiliser les bibliothèques publiques comme centre d'information communautaire parce que:

a) les bibliothèques, traditionnellement, ont toujours recueilli et organisé l'information:

b) les bibliothèques sont des «terrains neutres» qui visent à servir tous les segments de la population et ce, gratuitement:

c) les bibliothécaires ont déjà un certain entraînement pour interpréter, référer s'il y a lieu. ou répondre habilement et avec tact aux questions:

d) l'information communautaire dont on a besoin existe déjà, la plupart du temps, dans les collections et les dossiers des bibliothèques publiques. La collection au complet peut également devenir une source d'information:

e) dans les contextes urbain et rural, les bibliothèques publiques sont généralement très présentes, la communauté leur fait confiance et les respecte. Les heures d'ouverture peuvent s'étendre aux samedis, aux soirées, aux jours fériés et aux dimanches:

f) l'inclusion de services d'information et de référence dans les programmes de bibliothèques publiques permettrait de regrouper, ensemble sous un même toit, l'organisation et la diffusion de programmes traditionnels des bibliothèques, d'information gouvernementale, de services de référence et d'information communautaire.

En 1978, une recherche effectuée par le Bureau of Municipal Research de Toronto arrivait aux mêmes conclusions ${ }^{28}$. Elle souhaitait également qu'on confie aux bibliothèques publiques l'information communautaire et fournissait à peu près les mêmes raisons que Bowron trois ans plus tôt. Elle signalait en outre quelques avantages supplémentaires: d'après les auteurs, le
23. Edgar S. Warner, Information needs...

24. M. Kochen and J.C. Donohue. Information for the community. Chicago. American Library Association, 1976. 282 p.

25. Clara S. Jones, Public library..., Dorothy Turick, Community information...: M.R. Owens and M. Braverman. The public library and advocacy: Information for survival. New York. Columbia University. Teachers College. 1974.
26. R.C. Baetz, «Libraries and community information services combined under culture and recreation's information access", Ontario Library Review, vol. 64, no. 1 (1980), 4-5.

27. Albert Bowron. The Ontario public library: review and reorganization. Toronto. Information. Media and Library Planners. 1976. $184 \mathrm{p}$.

28. Bureau of Municipal Research. The public library as community information center. Toronto. 1978. 
personnel des bibliothèques est en fait composé de spécialistes de l'information, ce qui devrait faciliter les choses. De plus, ajoutaient-ils, grâce à leurs réseaux de succursales, les bibliothèques publiques peuvent devenir les agences les plus attentives aux besoins de leurs communautés respectives.

Au Québec, le rapport Aubry-Denis sur les bibliothèques publiques de l'île de Montréal recommandait qu'on établisse autant que faire se peut des centres d'information communautaires dans les bibliothèques publiques ${ }^{29}$.

\section{Situation au Québec et les voies d'avenir}

Yvon-André Lacroix déclarait avec justesse, ily a quelques années: «II faut surtout que les bibliothécaires des bibliothèques publiques soient des vendeurs, d'excellents vendeurs... d'un produit aussi essentiel que l'eau et le pain ${ }^{30}$. II s'agit en effet de "vendre» les bibliothèques, pas seulement parce que nos emplois sont en jeu mais effectivement parce qu'elles sont essentielles à la société. En effet, tout le monde a des besoins d'information et la bibliothèque publique peut garantir ce droit à l'information. Comme plusieurs auteurs l'ont bien souvent écrit, elle est en quelque sorte « un des remparts de la démocratie», en autant cependant, que la population sache que la bibliothèque publique existe et qu'elle l'utilise régulièrement. Or, quand environ $80 \%$ de la population d'un pays n'utilise presque pas ses bibliothèques publiques, le rempart devient une mince clôture de broche.

Cependant, est-ce bien l'information que l'on tente de vendre dans les bibliothèques publiques du Québec? Si l'on passe en revue les différentes publications du ministère des Affaires culturelles ces dernières années, notamment les rapports annuels de la Commission des bibliothèques publiques, le mot « information » n'y apparaît pour ainsi dire jamais. Les mots qu' on utilise lorsqu' on veut parler de l'activité principale des bibliothèques publiques sont "lecture» et «livre». Une importante brochure publiée en 1980 et destinée aux municipalités pour les inciter à développer leurs services de bibliothèque s'intitule: $L a$ bibliothèque publique: le livre partout et pour tous. Or, il s'agit là d'une vision très restreinte des possibilités d'une bibliothèque publique.

Les principes de base du marketing enseignent qu'une vision trop restrictive du produit conduit à l'échec ${ }^{31}$. Ainsi, les firmes modernes s'efforcent maintenant de vendre le transport plutôt que le train, la beauté plutôt que les cosmétiques, etc. De même les bibliothèques publiques doivent s'efforcer de vendre plus que le livre ou la lecture si elles veulent continuer de se développer. Or. le concept de bibliothèques publiques comme centre d'information communautaire ou populaire répond à cet impératif. II implique plus que le concept «lecture» car il oblige, par exemple, à développer un service de référence complet, y compris un service d'information par téléphone. II amène la clientèle à penser que, quoiqu'elle fasse, elle a besoin de la bibliothèque publique: si elle part en vacances, elle peut venir se documenter avant de partir; si elle a des ennuis avec sa voiture elle peut venir consulter un traité de mécanique; si elle veut réparer sa toiture, elle a tout ce qu'il faut pour savoir comment faire: si elle veut une recette de cuisine quelconque, elle peut toujours téléphoner pour la connaître; si elle cherche une gardienne pour ses enfants, elle pourra appeler, etc. Ainsi, si l'usager potentiel n'est pas intéressé par le «livre» ou la «lecture», il pourra quand même utiliser la bibliothèque. D'où un plus grand appui de la population et, par conséquent, de meilleures subventions, car, comme l'écrit Blaise Cronin: "To be is to be seen $\gg 32$

Donc. l'information est beaucoup plus facile à vendre que la lecture et les bibliothèques publiques du Québec devraient profiter de l'occasion. car il n'y a pas vraiment ici d'autres organisations capables de remplir ce rôle: du moins pas pour le moment. II existe, bien sûr. CommunicationQuébec, mais cet organisme gouvernemental se limite à diffuser de l'information en provenance des différents ministères du Québec ${ }^{33}$. Il y a aussi quelques centres d'information communautaires autonomes qui se sont constitués un peu partout au Québec devant la demande sans cesse croissante. Le plus important est sans doute le Centre de référence du grand Montréal, patronné par Centraide où l'on répond à plus de 60000 demandes d'information par année. Mais les centres sont plutôt marginaux, leur financement est d'ailleurs plus ou moins assuré d'année en année et il y a certainement place pour d'autres diffuseurs d'informations non spécialisés au Québec. Les bibliothèques publiques, comme nous l'avons démontré plus tôt, seraient l'endroit idéal autour duquel pourrait se bâtir ce réseau d'information populaire.

II y a cependant une réticence par rapport à ce nouveau rôle des bibliothèques publiques, réticence que nous arrivons mal à expliquer si ce
29. C. Aubry et L.G. Denis, Rapport de l'étude des bibliothèques publiques de la région de Montréal. Québec. Ministère des Affaires culturelles, 1976, p. 232.

30. Yvon-André Lacroix, «Présent et futur du réseau des bibliothèques publiques au Québec». Documentation et bibliotheques, vol. 25, no 4 (1979), 187
31. Theodore Levitt, «Un classique du management: Marketing Myopia ». Direction, vol. 150 (mai 1968), 546-573

32. Blaise Cronin, "To be is to be seen", New Library World, vol. 31. no. 957 (1980), 52-54

33. R. Vaillancourt et $D$. Gosselin. "Une banque de renseignements au service des citoyens québécois". Revue canadienne des sciences de l'information, vol. 1, no 1 (1976). 43-50. 
n'est par ces deux raisons : d'une part, un manque de personnel professionnel qui limite le développement et l'innovation et, d'autre part, une certaine confusion quant aux buts et objectifs des bibliothèques publiques.

Nous savons tous combien le réseau québécois des bibliothèques publiques souffre d'un manque de personnel compétent depuis toujours. Qu'il suffise de mentionner qu'il n'y a au Québec que 382 bibliothécaires et bibliotechniciens dans ce secteur ${ }^{34}$ alors que les bibliothèques publiques de l'Ontario, en termes de bibliothécaires professionnels seulement, peuvent compter sur plus de 1000 personnes. L'écart est si énorme qu'on ne pense même pas un jour pouvoir le combler même si les normes du ministère des Affaires culturelles mentionnent toujours qu'il faut un bibliothécaire par 6000 habitants ${ }^{35}$.

Nous croyons que ce manque de personnel professionnel nuit énormément au développement des bibliothèques publiques au Québec. En effet, dans la majorité des cas, les bibliothécaires déjà en place doivent mettre les bouchées doubles pour simplement arriver à assurer un service de base. Il faut d'ailleurs louer leur courage de pouvoir travailler si efficacement dans de si mauvaises conditions. Malheureusement, on peut difficilement leur demander de créer indéfiniment de nouveaux programmes, d'innover, de développer de nouveaux services, etc. Ce type d'opérations demande beaucoup de recherche et de planification que bien souvent le personnel en place ne peut mener parce que trop pris par l'administration au jour le jour de leur bibliothèque.
Si l'on veut que les bibliothèques publiques se développent et instaurent des services d'information populaires, il faudra donc travailler le dossier du personnel dans les prochaines années. II s'agit là d'une question primordiale.

Un autre malaise chronique qui, à notre avis, affecte douloureusement les bibliothèques publiques du Québec. c'est la question des buts et des objectifs. En effet, nous n'avons pas au Québec d'équivalent au "Mission Statement» des bibliothèques publiques américaines ${ }^{36}$, ni une loi régissant les bibliothèques publiques de façon aussi détaillée que dans d'autres états. II serait temps de se pencher sérieusement sur le sujet car l'avenir des bibliothèques publiques en dépend. En effet, sur quelle orientation faudrait-il mettre la priorité dans les prochaines années: une orientation «culturelle», axée davantage sur le livre et la lecture, plus susceptible de n'amener à la bibliothèque qu'un segment particulier de la population, c'est-à-dire une certaine "élite», ou une orientation plus «populaire», axée davantage sur l'information, plus susceptible à notre avis de rallier un plus grand nombre d'adhérents et qui englobe de toute façon la première orientation?

A notre avis, le choix est clair; il faut privilégier l'approche la plus englobante, celle qui met en valeur d'abord et avant tout le rôle d'information des bibliothèques publiques, tout en conservant leur rôle culturel. Actuellement, c'est l'approche qui répond le plus aux besoins de la société moderne, celle qui est la plus susceptible de conférer à la bibliothèque publique l'importance et le caractère essentiel qu'elle mérite.
34. D'après une enquête téléphonıque réalisée en janvier 1982 dans le cadre d'une recherche plus large: Réjean Savard. A Study of Professionalısm in Relation to Profes. sional Practice Attitudes and Values. Thèse de Ph.D.. Toronto. University of Toronto. 1983.
35. Québec. Ministère des Affaires culturelles. Normes pour les bibliothèques publiques, Québec, 1981, $56 \mathrm{p}$.

36. Public Library Association. The publıc library mission statement and its imperatives for service. Chicago. American Library Association, 1979. 